\title{
INTERVIEW WITH SUNEET MITTAL, MD, FACC, FHRS
}

\section{ANGELINA WAGNER}

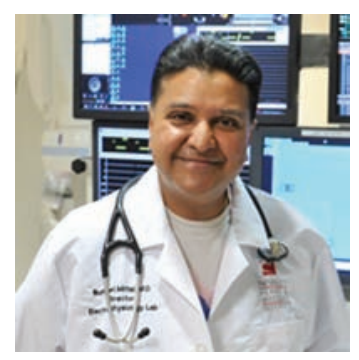

\section{Introduction}

Remoting monitoring has changed cardiovascular implantable electronic devices (CIEDs) patient care. Studies have shown remote monitoring benefits can include earlier detection of arrhythmias and device abnormalities, easier detection of heart failure risk, improved longterm survival, and reductions in recurrent hospitalizations without active patient participation. CIEDs with wireless remote monitoring are at the forefront of technologies because of the vital data that can be acquired outside of the traditional health care setting.

Electrophysiologists have been testing the efficiency and safety of remote monitoring in clinical and pre-clinical trials. The newest generations of cardiac devices from most major manufacturers are now compatible with automated, Web-based, remote monitoring systems. Despite the proven benefits, adoption of remote monitoring for cardiac devices has been difficult for some practices. Remote monitoring represents a shift in the way patient care is performed. New workflows and multiple systems management are required. Although these barriers might present themselves, the keys to implementing successful remote monitoring are having clear strategies and tactics for maintaining long-term results, and educating patients.

Dr. Suneet Mittal is Associate Chair of Cardiovascular Services for Valley Medical Group, Medical Director of The Valley Hospital's Snyder Center for Comprehensive Atrial Fibrillation, and Director of Cardiac Research for Valley Health System. Dr. Mittal is also Director of Electrophysiology for The Valley Hospital in New Jersey and New York.

He has been an author of over 106 publications in indexed journals, about 175 abstracts, 3 books and 16 electrophysiology book chapters. Dr. Mittal has presented at numerous national and international conferences as an invited speaker. As a member of several professional organizations and societies, he also serves on the Editorial Board of several electrophysiology and cardiology journals. Dr. Mittal's active research interests include the evaluation and management of patients with atrial fibrillation, the evaluation and management of patients with unexplained syncope, the role of implantable monitors in patients with known or suspected arrhythmias, and overcoming barriers to remote patient monitoring

We speak with Dr. Mittal, Associate Chair of Cardiovascular Services for Valley Medical Group about the cuttingedge clinical research in remote monitoring.

Wagner: How have cardiovascular implantable electronic devices with remote monitoring optimized patient outcomes?

Mittal: In the United States, wireless remote monitoring of cardiac implantable electronic devices (CIEDs) has been available for more than a decade. We now recognize that remote monitoring allows earlier detection of battery depletion, issues related to the integrity of implanted leads, changes in percentage of ventricular pacing, atrial and ventricular arrhythmias, as well as impending decompensation of congestive heart failure. There are data to suggest that this in turn results in decreased risk of stroke, inappropriate as well as appropriate defibrillator shocks, cardiovascular hospitalizations, and even mortality. Furthermore, remote monitoring may reduce costs related to healthcare utilization.

\section{Wagner: In your research, what changes have you seen in patient care for those using remote monitoring?}

Mittal: The biggest change that I have observed is that remote monitoring has now become the standard of care for CIED follow-up. Historically, CIED patients were asked to present for in-office device follow-up every 3-12 months, depending on the type of device, the duration since device implantation, and the overall wellbeing of the patient. Today, remote monitoring has supplanted this calendar based method for in-office follow-up. Specifically, patients are remotely monitored every 1-3 months and undergo in-office follow-up only annually, unless an alert condition is detected. 
Wagner: What data has multiple randomized clinical trials, as well as analysis of large registry data sets, provided in the way of strategies that can be incorporated in remote monitoring?

Mittal: Randomized clinical trials have conclusively demonstrated that remote monitoring facilitates earlier detection of battery depletion, lead related issues, changes in percentage of right and left ventricular pacing and detection of atrial and ventricular arrhythmias. These trials have also shown that remote monitoring is associated with reduced healthcare utilization. However, the impact on mortality remains undefined. Some trials have shown a significant reduction, whereas others have not. It should be noted, however, that these prospective trials have had relatively small sample size, a variable mix of CIED types, differing follow-up durations, the type of remote monitoring system varied, and adherence to remote monitoring was not always assessed or reported. In contrast, the large registry data sets have consistently shown an association between remote monitoring and reduced healthcare utilization as well as survival, irrespective of CIED type, especially when patients had a high degree of adherence to remote monitoring.

Wagner: With your participation on the 2015 HRS Expert Consensus Statement on Remote Interrogation and Monitoring for Cardiovascular Electronic Implantable Devices, what were the key statements from this consensus? Also, has it provided a new standard of care?

Mittal: In my opinion, the 2015 HRS Expert Consensus Statement of Remote Interrogation and Monitoring for Cardiovascular Electronic Implantable Devices does establish a new standard of care for these patients. I believe that the following 3 recommendations represent the key statements from this consensus document:

1. A strategy of remote CIED monitoring and interrogation, combined with at least annual in-person evaluation, is recommended in preference to a calendar-based schedule of in-person CIED evaluation alone (Class I; LOE: A)

2. All patents with CIEDs should be offered remote monitoring as part of the standard follow-up management strategy (Class I; LOE: A)

3. It may be beneficial to initiate remote monitoring within 2 weeks following CIED implant (Class IIA; LOE: C)

\section{Wagner: What are the benefits of remote monitoring for CIED patients?}

Mittal: There are many benefits to remote monitoring in CIED patients. First, there is the peace of mind for the patient knowing that the device is being interrogated on a daily or weekly basis to determine whether anything requires immediate attention. Second, earlier attention to these "alert" conditions may prevent the patient's overall condition from deteriorating. Lastly, remote monitoring provides the patient with a convenient option for device follow-up; the patient is certainly saved the time and expense of having to go in for a routine in-office evaluation of a normally functioning CIED.

Wagner: How will remote monitoring cause physicians to reevaluate the relative value of routine in-person device interrogations?

Mittal: It is important to recognize that the guidelines do not advocate elimination of in-person device interrogations. In fact, they continue to recommend an annual in-person evaluation to assess the device along with the device pocket and patient. However, remote monitoring certainly eliminates the need for a calendar based quarterly device evaluation. Most device checks show the system to be functioning normally and the patient to be free from any significant arrhythmia; this information can now be retrieved easily through remote monitoring, thus enhancing the convenience for the patient. In office evaluations can now be better targeted towards addressing those conditions that generate an alert(s).

\section{Wagner: What are the potential barriers to remote monitoring?}

Mittal: We have identified several barriers to remote monitoring. First, many physicians who follow CIED patients do not yet fully appreciate the value proposition of remote monitoring and continue to favor calendar based in-office device interrogations. Thus, greater education is still necessary to the community of physicians who follow CIED patients. Second, better efforts are needed to educate patients on the value of remote monitoring. An additional necessary step for patients is "plug and play" remote monitoring systems that simply require connection to a power outlet and are then capable of cellular communication over a GSM system. To date, the need for an analog phone line or having to incur the expense of installing alternative methods for data communication have hindered attempts to get all CIED patients to enroll in remote monitoring. Once enrolled, many patients do not remain adherent to remote monitoring. Recent data show the relationship between greater adherence and improved survival; thus, patients need to be educated on the health benefits of high compliance with remote monitoring. Lastly, physicians and their practices need better support in helping establish the infrastructure that is necessary to initiate and maintain an active remote monitoring program. 


\section{Wagner: What technical procedural skills should physicians have for remote monitoring?}

Mittal: The 2015 HRS Expert Consensus Statement of Remote Interrogation and Monitoring for Cardiovascular Electronic Implantable Devices provides clear guidance on this matter. Specifically, it states that "physicians who oversee remote interrogation and remote monitoring programs should have the skills to interpret all CIED data and intracardiac electrograms, as well as troubleshoot and treat CIED related problems". The document further recommends that "physicians responsible for supervising and reviewing remote interrogation and remote monitoring data possess board certification from either the American Board of Internal Medicine (ABIM) Clinical Cardiac Electrophysiology certification, American Board of Pediatrics Cardiology certification, or the International Board of Heart Rhythm Examiners (IBHRS) device certification.

Wagner: How do specific protocols for patient assessment and care play a key factor into educating patients about the benefits of remote monitoring?

Mittal: Patient education is critical to developing a successful remote monitoring program. In patients with a wireless CIED, the protocol for device follow-up will vary depending on whether the device is an implantable loop recorder (daily remote monitoring), the patient's congestive heart failure is being managed through device diagnostics (monthly remote monitoring), or whether remote monitoring is being for generalized device and patient monitoring (at least quarterly remote monitoring). Furthermore, the intensity of monitoring will vary based on the indication for monitoring. For example, a patient with history of cryptogenic stroke in whom a loop recorder is implanted for longterm ECG monitoring to exclude episodes of atrial fibrillation may need "tighter" monitoring than a patient with atrial fibrillation who has a single chamber pacemaker but is not pacemaker dependent.

\section{Wagner: What advancements in devices and remote monitoring do you see on the horizon?}

Mittal: There are several advancements that I see on the horizon in the field of remote monitoring of CIEDs. First, I think all CIEDs being implanted worldwide will be capable of wireless remote monitoring. Second, the patient's personal mobile phone will likely serve as the intermediary "base station", facilitating the transfer of diagnostic information from the device itself to a central monitoring station. Third, I think we will have more robust systems to alert patients as well as physicians when devices have stopped communicating with their remote monitoring systems. Finally, I think patients will have greater access to the diagnostic data stored in their device, thus paving a road to greater engagement with our patients.

Wagner: What improvements need to be made to speed access to new devices and support expansion of remote monitoring?

Mittal: To increase adoption of remote monitoring, the systems have to become easier for patients to use. Towards that end, base stations that only require the patient to plug them into a power outlet when they get home are essential. Information should flow automatically over GSM based cellular networks, with the provision of using WiFi when necessary. The elimination of analog phone lines, need for adapters, etc. is critical to enhancing adoption of remote monitoring. From a physician standpoint, development of strategies for more efficient data management are critical, including systems that seamlessly extract data from the devices and populate them within the patient's electronic health record.

\section{Wagner: Are there any additional thoughts you would like to add?}

Mittal: Thank you for the opportunity to participate in this dialogue on remote monitoring of CIEDs. I believe that this is truly the new gold standard for device management. However, adoption on the part of patients and physicians remains unacceptably low, for a variety of reasons. It is imperative that these issues be addressed so that all involved parties can maximize the clinical utility of remote monitoring. 